\title{
Innovative technologies for industrial sewage treatment
}

\author{
Ilyas Shigapov 1,*, Alesya Kadyrova ${ }^{2}$, Bahromjon Akhmadov $^{3}$, and Olga Krasnova ${ }^{4}$ \\ ${ }^{1}$ Technological Institute - branch of the Federal State Budgetary Educational Institution of Higher \\ Education "Ulyanovsk State Agrarian University named after P.A.Stolypin", Dimitrovgrad, \\ Ulyanovsk region, Russian Federation \\ ${ }^{2}$ PKIUGGT (Branch) of FGBOU VO MGUTU named after K.G. Razumovskiy (PKU), Dimitrovgrad, \\ Ulyanovsk region, Russian Federation \\ ${ }^{3}$ Tajik Agrarian University named Shirinsho Shotemur, Dushanbe, Tajikistan \\ ${ }^{4}$ DITI NIYAU MIFI, Dimitrovgrad, Ulyanovsk region, Russian Federation
}

\begin{abstract}
At present the Branch is working on the development of modern methods to improve the efficiency of water treatment and sewage treatment systems of industrial enterprises and enterprises processing agricultural products through the use of membrane technology.
\end{abstract}

Nowadays industrial effluents of the gas industry are cleaned using typical biotechnology in aerotanks, biofilters and oxytanks. In this regard, the actual efficiency of the treatment facilities is lower than the design: the average degree of cleaning is less than $65 \%$.

Most of the existing treatment facilities were built in the 60-70s and do not meet modern requirements for the quality of sewage treatment. At the same time, the construction and operation of sewage treatment plants requires considerable financial costs, so their effectiveness is largely determined by the use of new modern water treatment facilities and technologies.Recent advances in science and technology allow forrelatively low costs for a short time qualitatively improve the operation of many existing treatment facilities by the intensification of cleaning process based on modern technologies and replacing outdated equipment.

The choice of the water treatment process scheme and the efficiency of its operation depend on the amount and quality of waste water, the possibility and economic feasibility of extracting impurities, requirements for the quality of treated water, conditionsof aeration, the degree of saturation of waste water with oxygen, etc. Insufficient oxygen leads to a decrease in the oxidative capacity of organic substances and, as a result, an increase in the cleaning time and a deterioration in the quality of the purified water.

Today, most aeration stations use porous ceramic materials (filter plates, pipes), synthetic materials, slot and hole pipes as air dispersants. However, the difficulties associated with the operation of this equipment, the low utilization of air oxygen required the search for new technical solutions.

\footnotetext{
* Corresponding author: schigapov@mail.ru
} 
One of the promising methods of intensification of biological and flotation treatment of waste water, which can increase the efficiency of treatment facilities and simplify their technological schemes, is the use of a developed membrane gas separation module that acts as an oxygen enrichment generator (up to 39-40\%) and a device of gas saturation (oxygenator) (Figure 1.).

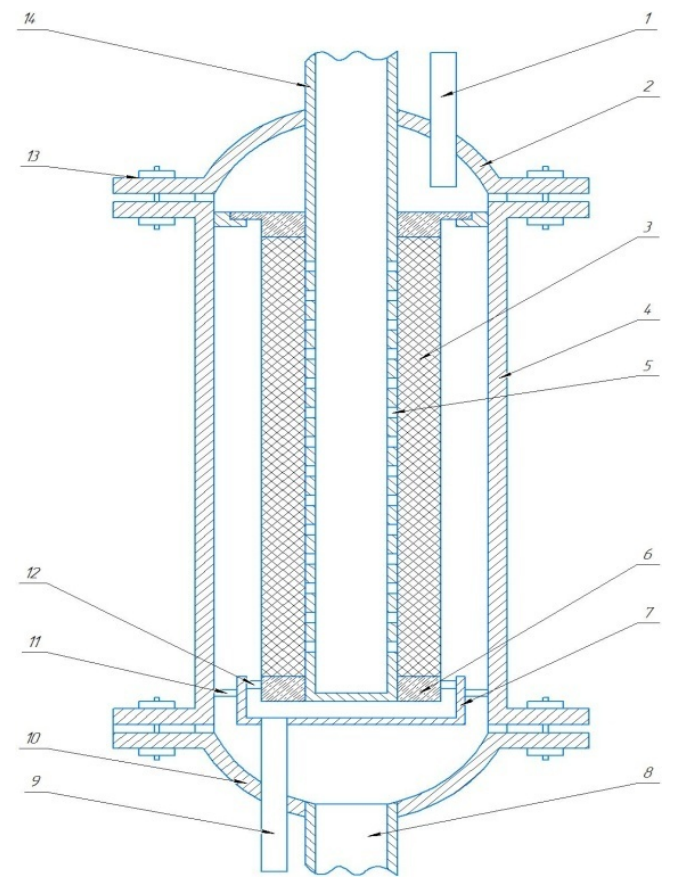

Fig. 1. Membrane gas separation module: 1 - branch pipeof gas supply; 2 - the first cover; 3 - hollow fiber membrane package; 4 -body ; 5 - hollow perforated core; b - glue unit; 7 - collectorof lean gas mixture; 8 - discharge branch pipeof oxygenated water; 9 - discharge of lean gas mixture; 10 - second cover; 11 - radial prop; 12 - sealing rings; 13 - seal; 14 - branch pipeof fluid supply

The module may also function as dispersing facilitiesto provide a microbubble gasliquid environment, as result creating the largest contact area of the gas and liquid phases per unit volume.

On the basis of the studies, a scheme was developed for the inclusion of membrane gas separation facilities in the chemical-fleet cleaningsystem of industrial effluents (Fig. 2). There are two variants for operation of the gas separation unit [1,2].

Variant 1. The gas separation unit is used as dispersing facilities. In order to intensify the treatment of industrial effluents, part of the water treated in the flotator is pumped $\mathrm{H} 1$ to the membrane hollow fiber module (valves BH4 and BH6 are opened, BH5 is closed), where at the same time compressor $\mathrm{K}$ is pumpedatmospheric air through air filter (valve $\mathrm{BH} 1$ is closed, BH2 is opened).

Water repleted with air enriched with oxygen up to 39-40\%, after pressure decrease on throttling device throttling device 1 directs to the pipeline that supplies the effluents to the flotator.

Research have shown that under certain pneumohydrodynamic conditions, there is formeda microbubble gas-liquid environment with bubble sizes of 10-20 micron. High volume content of microbubbles in environment is achieved - up to $8-10 \%$ of volume with dissolved oxygen content of $19-20 \mathrm{mg} / \mathrm{l}$ (240\% saturation), which is many times higher than oxygen content in source water. Microbubble gas-liquid environment has large 
interfacial surface and due to collective hydrodynamic interaction of microbubbles has properties of three-dimensional diffusion, that together allows to increase intensity of flotation treatment of waste water from microdisperse impurities substantially[3,4].

Variant 2. The gas separation unit is used as a generator for producing air enriched with oxygen up to $39-40 \%$.

Atmospheric air is supplied by compressor K through air filter (valve BH1 is closed, VHZ is opened) to membrane gas separation unit for oxygen enrichment [5]. Enriched air is directed to the mixing chamber (VHZ valve is opened) where at the same time the pump $\mathrm{H} 1$ pumps purified water (BH6, BH5 gates are opened, $\mathrm{BH} 4$ is closed). Further, the waterair mixture is pumped through the throttling device 2 into the pipeline which is to be treatedwith industrial effluents, as a result the oxidativeprocesses of the treated effluents are intensified.

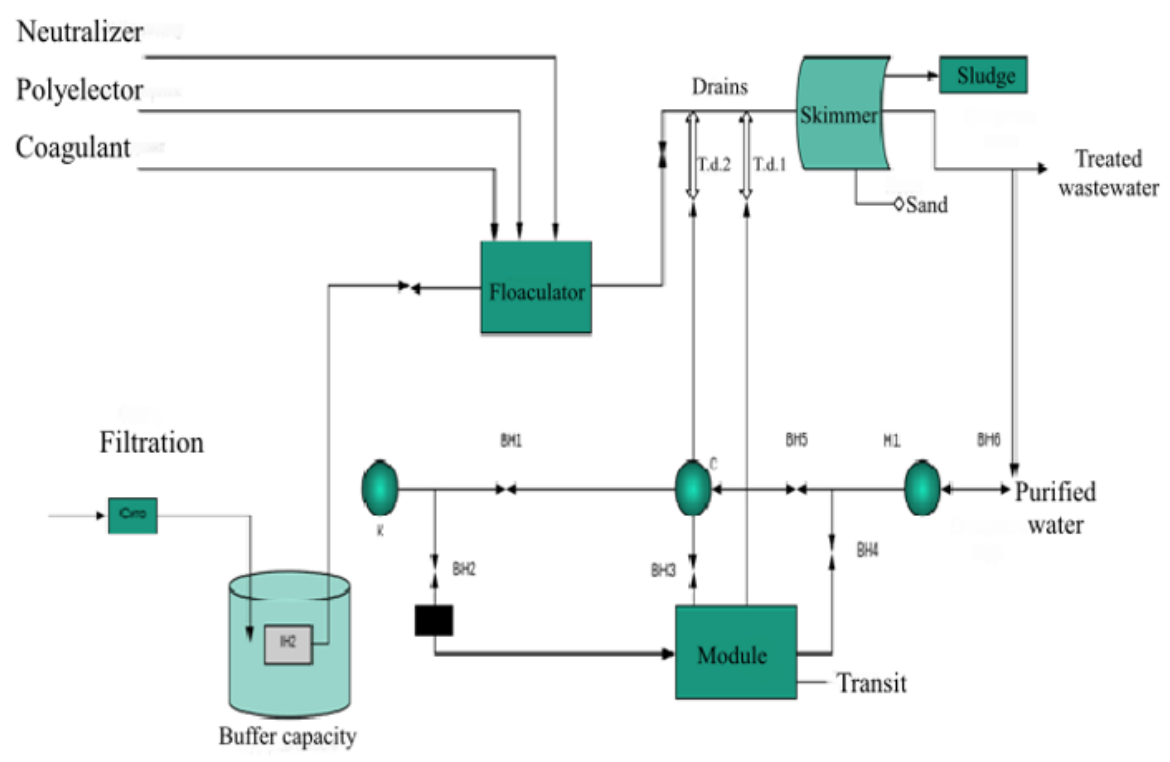

Fig. 2. Scheme of inclusion of the membrane gas separation unit in the effluent treatment system: 1 -1 variant; 2-11 variant; $\mathrm{N}$ - the pump; T.d. - throttling device; $\mathrm{C}$ - mixing chamber.

The use of membrane technology in the chemical flotation cleaningsystem made it possible to reduce the cleaning time, increase its quality, save expensive chemical reagents, which ensures a reduction in the cost of cleaningby 30-35 \% or more [6, 7].

The introduction of membrane technology offers great opportunities to increase the productivity of industrial units, reduce specific fuel consumption, reduce the amount of harmful emissions into the environment, that will ultimately help to solve the environmental problems of the gas industry and agriculture. At the same time, the usefulness of membrane separation and cleaningof mixtures in each case should be determined on the basis of the results of the feasibility studies, taking into account the availability of the required separation material, its properties and cost, as well as the degree of excellence of alternative technological processes. 


\section{References}

1. Gubeidullin H.H., Shigapov I.I., Kafiyatullova A.A. Tubular textile aerator for sewage treatment//Rural machine operator. - 2014. - No. 2. - P. 28-29.

2. Gubeidullin H.H., Shigapov I.I., Porosyatnikov A.V., Lukoyanchev S.S. New types of textile filters//Rural machine operator. - 2015. - No. 6. - P. 32-33.

3. Gubeidullin H.H., Shigapov I.I., Porosyatnikov A.V., Lukoyanchev S.S., Kamaldinova O.S. Study of the air breathabilityof porous partitions of tubular textile filters//News of higher educational institutions. Textile technology. 2015. No.5 (358). P. 244-247.

4. Gubeidullin, H.H. Tubular textile filters for milk cleaning/H.H. Gubeidullin [others ]//Rural machine operator. - 2011. - No. 1. - P. 28-29.

5. Shigapov I.I., Porosyatnikov A.V., Lukoyanchev S.S., Kadyrova A.M., Krasnova O.N. Bubbling aerators for waste water treatment of stock-raising farms.//Rural machine operator. 2018. No 6. P. 28-29.

6. Gubeidullin H.H., Panin I.N., Shigapov I.I., Porosyatnikov A.V. Development and study of filter partitions of flat and tubular textile filters.//News of higher educational institutions. Textile technology. 2015. No.1 (355). P. 159-164.

7. Gubeidullin H.H., Shigapov I.I., Porosyatnikov A.V., Lukoyanchev S.S., Kamaldinova O.S. Hydraulic properties of porous partitions of tubular textile filters.//News of higher educational institutions. Textile technology. 2015. No.5 (358). P. 215-219 